\title{
Systematic Spectroscopic Observations on Small Telescopes: Past and Future Research of Stellar Kinematics
}

\author{
M.E. Sachkov \\ Institute of Astronomy, 48 Pyatnitskaya Str., Moscow 109017, Russia \\ E.V.Glushkova, \& A.S.Rastorguev \\ Sternberg Astronomical Institute, 13 Universitetsky Ave., Moscow \\ 119899, Russia
}

\begin{abstract}
We have used a CORAVEL-type spectrometer on small telescopes (70-cm, Moscow observatory, Russia; 60- and 100-cm, Simeiz observatory, Crimea, Ukraine and some others) for systematic observations of northern sky stars, since 1987 (Rastorguev \& Glushkova, 1997; Gorynya et al., 1998). The typical accuracy of a single measurement is about $0.5 \mathrm{~km} / \mathrm{s}$. The limiting magnitude is 14 in V-band. The young Galaxy disk population, metal-poor stars and Kaptein selected areas are the important parts of our program. In this paper we present new results on galactic kinematics and our future program.
\end{abstract}

The young disk population, which includes neutral hydrogen, HII-regions, OB-associations and supergiants, classical Cepheids and young open clusters, is characterized by small velocity dispersions $(6-15 \mathrm{~km} / \mathrm{s})$ and, consequently, by small rotational lag relative to the LSR. This explains why such objects are used to study the rotational law of the galactic disk. Most studies of Population-I kinematics are based on radial-velocity data for objects with well-established and homogeneous distance scales.

We have summarized the information about our own observations in Table 1. Our measurements of radial velocities, taken together with precise absolute proper motion measurements (HIPPARCOS, 1997), have enabled the detailed investigation of the space velocity field of young objects. The most reliable results have been derived for open clusters and Cepheids:

1. The Galactic rotation curve and the kinematical parameters:

$\left(\Omega_{0}, \Omega_{0}^{\prime}, \Omega_{0}^{\prime \prime}\right)=\left(28.0 \pm 1.3,-4.67 \pm 0.24 \mathrm{kpc}^{-1}, 1.16 \pm 0.27 \mathrm{kpc}^{-2}\right) \mathrm{km} / \mathrm{s} / \mathrm{kpc}-$ the disk angular speed and its derivatives;

$V_{0}=(6.9 \pm 3.2,11.1 \pm 2.2,6.9 \pm 1.3) \mathrm{km} / \mathrm{s}$ - the solar velocity with respect to the local centroid, including the noncircular motion of the centroid;

$\left(\sigma_{U}, \sigma_{V}, \sigma_{W}\right)=(12.1 \pm 1.4,8.0 \pm 1.0,7.2 \pm 1.3) \mathrm{km} / \mathrm{s}$ - the components of the velocity dispersion tensor; ations;

$\left(f_{R}, f_{\theta}\right)=(-6.8 \pm 2.7,1.4 \pm 1.9) \mathrm{km} / \mathrm{s}$ - the amplitudes of systematic devi-

$\left(i, \chi_{0}\right)=(5.8 \pm 0.6,86 \pm 22)^{0}-$ pitch angle and phase angle of the Sun respectively. 


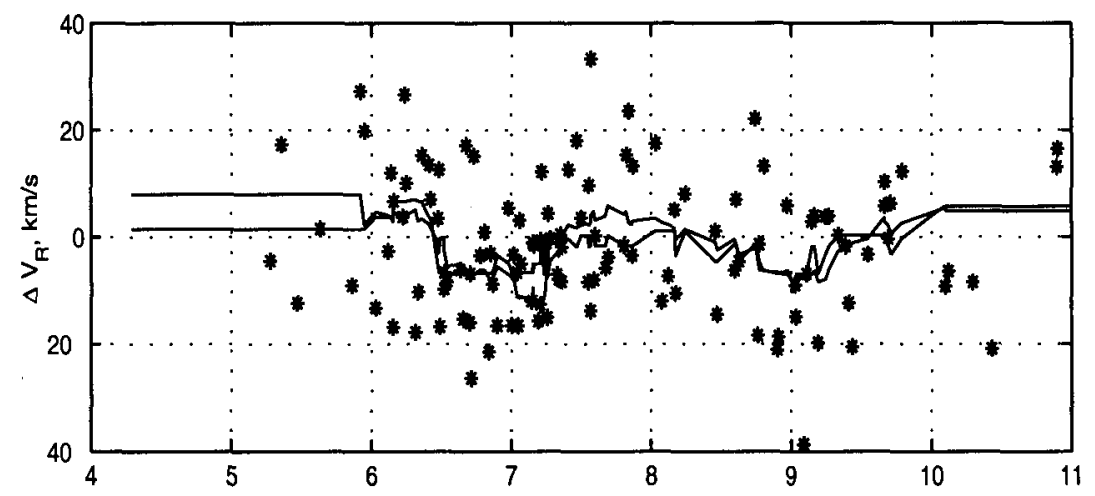

Figure 1. Radial residual velocity, $\Delta V_{R}$, from purely circular motions (in $\mathrm{km} / \mathrm{s}$ ) of young open clusters and Cepheids as a function of galactocentric distance $R(\mathrm{kpc})$ (positive from the galactic center).

2. The radial systematic deviation with an amplitude of the order $7 \mathrm{~km} / \mathrm{s}$ can be considered as an argument for the wave nature of the galactic spiral arms (see Fig.1).

3. The statistical-parallax method applied to classical Cepheids with periods $>10^{d}$ leads to an LMC distance modulus of less than 18.40, which agrees, within the errors, with the short distance scale.

Table 1. Radial velocity measurements

\begin{tabular}{llll}
\hline Objects & $\begin{array}{l}\text { Number of } \\
\text { measurements }\end{array}$ & $\begin{array}{l}\text { Number of } \\
\text { objects }\end{array}$ & Aim of research \\
\hline Open clusters & 1400 & 642 for 43 clusters & Kinematics \\
Cepheids & 7211 & 144 & Kinematics \\
Late type supergiants & 1080 & 300 & Kinematics \\
Stars in & & & \\
Kaptein selected areas & 1300 & 1150 & {$[\mathrm{Fe} / \mathrm{H}]$} \\
Metal-poor stars & 120 & 100 & {$[\mathrm{Fe} / \mathrm{H}]$} \\
\hline
\end{tabular}

Acknowledgments. We are grateful to M.V.Zabolotskikh for useful discussions and comments, to Drs. N.N.Samus and N.A.Gorynya for taking part in our observations and to Mrs. Sachkova for the assistance. This study was supported, in part, by the Russian Foundation for Basic Research.

\section{References}

ESA, 1997, The Hipparcos and Tycho Catalogues, SP-1200.

Rastorguev, A.S., Glushkova, E.V. 1997, Astronomy Letters, V. 23, P.1.

Gorynya, N.A., Samus, N.N., Sachkov, M.E., Rastorguev, A.S., Glushkova, E.V., and Antipin, S.V. 1998, Astronomy Letters, V. 24, P. 815. 